\title{
Lifetime Planning for TDMA-Based Proactive WSN with Structured Deployment
}

\author{
Sebastià Galmés \\ Universitat de les Illes Balears \\ Cra. de Valldemossa, km. 7.5 \\ 07122 Palma, Spain \\ +34-971172989
}

sebastia.galmes@uib.es

\begin{abstract}
It is commonly accepted that the primary design goal in wireless sensor networks is the reduction of energy costs. Thus, the concept of lifetime planning is prioritized over capacity planning when dealing with such networks instead of the traditional ones. This paper offers a systematized treatment of the lifetime of TDMA-based proactive sensor networks with structured deployment. This is the case of many environmental networks, which are devoted to the regular monitoring of some physical or even chemical variables, for scientific study and/or for taking appropriate control actions. On the other hand, sensor nodes in structured deployments are located at strategic positions that may not be as close to each other as in random and massive deployments. Thus, in the second part of this paper, a costeffective mechanism that consists of inserting pure switching nodes in some links is proposed, as a way of enhancing lifetime by alleviating the severe effects of the path loss exponent in the radio propagation model.
\end{abstract}

\section{Categories and Subject Descriptors}

C.2.1 [Computer-Communication Networks]: Network Architecture and Design-distributed networks, packet-switching networks, wireless communication.

\section{General Terms \\ Design.}

\section{Keywords}

Time Division Multiple Access (TDMA), data aggregation.

\section{INTRODUCTION}

Wireless sensor networks can be broadly categorized as reactive or proactive [1]. Reactive networks are event-driven, that is, they are designed to detect the occurrence of some pre-specified event and automatically report it to the sink or base station. So, communication is triggered by the event, and only nodes within the event area participate in the communication process. This is the case of many detection or alarm-based real-time applications.

Permission to make digital or hard copies of all or part of this work for personal or classroom use is granted without fee provided that copies are not made or distributed for profit or commercial advantage and that copies bear this notice and the full citation on the first page. To copy otherwise, or republish, to post on servers or to redistribute to lists, requires prior specific permission and/or a fee.

LANC'07, October 10-11, 2007, San José, Costa Rica.

Copyright 2007 ACM 978-1-59593-907-4/07/0010_..\$5.00.
Alternatively, in proactive sensor networks, communication is triggered by nodes, which take readings of their local environment and transmit them to the base station. All nodes participate in the communication process by continuously sampling the environment and reporting the corresponding information towards the base station. This is the case of many environmental applications, where some physical or even chemical variables, such as temperature, humidity, solar radiation, level of contaminants... must be monitored and reconstructed in time and space at the data management center, for scientific study and/or for taking appropriate control actions (normally at larger timescales than in reactive networks). Some interesting examples are found in [2]. A good survey of applications for sensor networks is provided in [3].

It is of primary importance that protocol design takes into account the above classification. For instance, while typically the traffic generated by reactive networks is sporadic and of bursty nature, the traffic generated by the proactive ones takes the form of a continuous flow. And from the point of view of energy consumption, there are also differences that deserve attention. Particularly, in reactive networks, the energy consumption caused by the sensing activity can achieve significant levels, because nodes must continuously be aware of the environment in order to rapidly detect unexpected or sporadic events. Also, the potential energy waste in communication due to idle listening becomes relevant. Thus, the design of protocols for reactive networks must focus on duty-cycling both the sensing and communicating activities in order to get a satisfactory balance among coverage, real-time responsiveness and network lifetime.

Alternatively, in proactive networks the energy cost of instantaneously sense the environment is much less than the energy required for communicating the corresponding sample. Thus, the analysis of lifetime for such networks can focus almost exclusively on the communication aspect. In addition, proactive sensor networks can take advantage of the fact that both the sensing and communicating activities are triggered by nodes themselves, thus becoming predictable. This is because such predictability makes contention-free protocols especially well suited for managing the packet flow, and since usually these protocols are TDMA-based, nodes can easily switch to sleep mode for all their non-assigned slots. The issue of duty-cycle reduction and other details about protocol design for proactive networks are treated in the next section.

Another important design issue is network deployment. As stated in [4], while random deployment is an appealing approach for some applications over inaccessible or impractical regions, many current sensor networks are deployed in a structured manner, sometimes by careful hand placement of the sensor nodes at 
strategic positions. These positions may not be necessarily very close to each other as it happens with random deployments. It is also common in proactive networks that data are routed through predetermined paths giving rise to static multi-hop routing schemes [5]. Certainly, such schemes suffer from the most popular node problem, since the node or nodes closest to the sink have to forward many packets. However, it is also true that this problem can be alleviated by aggregating redundant data en route to the sink, thus reducing traffic and saving energy [5]. The introduction of data aggregation techniques is briefly considered at the end of Section 6.

As stated above, the resulting single-hop distances in structured networks may become significant (up to a few hundreds of meters). For such distances, the path loss exponent in the radio propagation model causes severe signal attenuation. Thus, an additional lifetime enhancing mechanism considered in this paper consists of strategically inserting pure switching nodes in the network, in order to reduce the communication range of at least the regular nodes with largest duty cycle. Note that products offered by several manufacturers allow for an easy and costeffective implementation of this strategy, since they follow a modularized architecture where the sensing unit can be detached from the mote to easily convert the node into a pure switching device.

The purpose of this paper is twofold. First, a systematized analysis of energy consumption and lifetime of TDMA-based structured proactive sensor networks with multi-hop static routing is performed. Next, the effects of inserting pure switching nodes on network lifetime are also analyzed. While it might appear trivial the fact that introducing new hardware enhances lifetime, the way it is done and the quantitative effects need careful attention.

As stated before, an important application of proactive sensor networks is environmental monitoring. And one of the most prominent environmental monitoring applications is fire control in forests. As reported in [6], wildfires are present at all ecosystems of South American countries, extending from $12^{\circ} \mathrm{N}$ to $56^{\circ} \mathrm{S}$. The frequency, intensity and time distribution of such wildfires during the year are variable in response to diverse human and environmental factors. Among the latter, some examples are the occurrence of the El Niño event (although fortunately not always related to wildfires), droughts and, sometimes, lightning storms.

At the same time, the biggest tropical rainforest extension in the world can be found in the South American region, reaching almost 885 million ha. located in the Amazon basin, and another 85 million ha. in the system formed by the Orinoco and Parana catchments, globally representing $95 \%$ of the continent's total forest cover. A substantial part of tropical evergreen forests are located in Peru, Colombia, Ecuador, Venezuela, French Guiana, Guyana, Suriname and Bolivia, but the vast majority of these forests is located in Brazil. Also, temperate forests are located primarily in Argentina, Chile, Paraguay and southern Brazil, covering only $5 \%$ of the continent's forests.

Unfortunately, this natural heritage has experienced an increasing trend in the occurrence of wildfires during the last 25 years. As a sample, the dramatic values for the annual number of wildfires and burned area in South America from 1986 to 2004 are shown in Table 1 [6].
Table 1. Annual number of wildfires and burned area in South America between 1986 and 2004 [6]

\begin{tabular}{|c|c|c|}
\hline Year & Number of fires & Area burned (ha) \\
\hline 1986 & 13 & 58.00 \\
\hline 1987 & 8 & 15040.00 \\
\hline 1988 & 9 & 60927.00 \\
\hline 1989 & 65 & 13509.00 \\
\hline 1990 & 5201 & 45698.00 \\
\hline 1991 & 11279 & 9759804.00 \\
\hline 1992 & 11280 & 654224.00 \\
\hline 1993 & 7533 & 1861720.00 \\
\hline 1994 & 2339 & 1688040.00 \\
\hline 1995 & 11490 & 979165.00 \\
\hline 1996 & 11572 & 564674.00 \\
\hline 1997 & 66807 & 5585369.00 \\
\hline 1998 & 15877 & 1137305.00 \\
\hline 1999 & 43016 & 13592352.00 \\
\hline 2000 & 16401 & 2891799.90 \\
\hline 2001 & 17966 & 4888276.40 \\
\hline 2002 & 23519 & 2607460.46 \\
\hline 2003 & 29158 & 3667639.85 \\
\hline 2004 & 9191 & 430418.19 \\
\hline
\end{tabular}

Sensor networks constitute an alleviating strategy to the problem represented by Table 1 from the perspective of information and communication technologies. Certainly reactive implementations can be very useful in detecting emerging fires and reacting appropriately, but the magnitude of the problem suggests that the use of a proactive preventive network, capable of monitoring different environmental variables and detecting high-risk situations, is highly recommendable. This falls within the scope of this paper.

More specifically, the rest of this paper is organized as follows. In Section 2, the main issues regarding protocol design for proactive sensor networks are outlined. Section 3 focuses on the analysis of energy consumption and network lifetime. In Section 4 a numerical example is considered, which is validated through simulation. Section 5 deals with the insertion of pure switching nodes in the network as a way of lifetime enhancement. Scalability issues regarding this strategy are considered in Section 6. Finally, in Section 7, the main conclusions and suggestions for further research are drawn.

\section{DESIGN ISSUES FOR PROACTIVE SENSOR NETWORKS}

The predictability of the communication pattern generated by a proactive sensor network makes the use of contention-free 
protocols especially appropriate. As stated in [4], contention-free scheduled MAC protocols for multi-hop wireless networks date back to the early studies on packet radio networks in late eighties. These protocols consist of the pre-allocation of transmission resources to nodes in order to avoid conflicts. Such resources can take the form of time slots in TDMA (Time Division Multiple Access), frequency channels in FDMA (Frequency Division Multiple Access) or distinct codes/hopping sequences in CDMA (Code Division Multiple Access). Among these techniques, the most commonly adopted for sensor networks is TDMA. While TDMA was not designed to target energy conservation, it becomes a simple and efficient way to reduce the duty cycle of nodes in a sensor network. This is because nodes can autonomously switch to sleep mode whenever they are not scheduled to transmit or receive.

An inherent problem to TDMA access mechanisms in multi-hop networks consists of assigning time slots (channels) to nodes, in such a way that the interference constraint is satisfied. This requires that no nodes within two hops of each other use the same slot, in order to avoid hidden terminal collisions. As stated in [7], the problem of assigning a minimum number of channels to an arbitrary graph, while ensuring that the two-hop constraints are satisfied, is NP-hard. Nevertheless, following heuristic algorithms it is still possible to provide efficient solutions to this problem. Thus, protocol overhead operations related to TDMA-based access mechanisms focus mainly on the way channel assignment is performed. Once this is accomplished, regular data transfer takes place according to the adopted TDMA frame structure.

One approach to channel assignment in TDMA-based sensor networks is the Self-Organizing Medium Access Control for Sensor Networks (SMACS) proposed in [8]. As stated in [1] and [9], SMACS is a distributed protocol that combines neighborhood discovery with the assignment of TDMA schedules to nodes. The two operations are performed concurrently, in such a way that a channel is assigned to a link immediately after the link's existence is discovered. So, by the time all nodes hear all their neighbors, a connected network is established, with at least one multi-hop path between any pair of nodes. Since there is a potential for collisions with time slots assigned to neighbor links, in this protocol each link is negotiated at a different frequency, which is randomly selected from a large set of alternatives. This reduces the likelihood of collisions.

Another approach is the BFS/DFS-based scheduling [10]. Here, nodes constitute a data-gathering tree rooted at the sink, and a unique slot or set of unique slots are finally assigned to each node depending on the load it needs to carry, so there is no spatial reuse of time slots. In the breadth-first (BFS) version, contiguous time slots are assigned to each node, whereas in the depth-first (DFS) one, all slots along a route from a source node to the sink are contiguous (in general, only a subset of nodes are devoted to sensing the environment and behave as source nodes). As stated in [10], unless the energy cost of switching nodes from active to sleep and vice versa is significant, DFS is preferable because it ensures better performance metrics (packet dropping probability due to buffer overflow, end-to-end delay and throughput). Unlike the case of environmental applications considered in the present paper, in [10] a highly dynamic scenario consisting of an eventdriven target detecting application is simulated, in which an energy-aware dynamic routing scheme is adopted and combined with time slot re-assignment according to BFS or DFS.
A disadvantage of TDMA mechanisms is that they are not flexible enough to allow data traffic from nodes to change over time. To overcome this drawback, ReSync [11] provides a reservation-based scheme where nodes can declare their intention to transmit or not. This is performed on the basis of a distributed TDMA scheduling, where each node manages an autonomous sequence of time periods called epochs, whose length is constant across the network. Each node picks a regular time to periodically transmit its intent messages, at a rate of one intent message per epoch. In case the node has one or several packets to transmit, the transmission time is randomly selected within the next epoch and announced in the current intent message as a relative rather than absolute time interval.

Another protocol is the Traffic-Adaptive Medium Access (TRAMA) [12]. Here, transmission schedules are created by nodes in a distributed manner and on an on-demand basis. It is assumed that all nodes are synchronized, and time is divided into a random access period and a scheduled-access period. The random access period is used by nodes to broadcast neighborhood and schedule information, which is in turn used by themselves to execute a distributed scheduling algorithm that allows to determine for each time slot of the scheduled-access period the nodes that can transmit, receive or go into sleep mode.

A common characteristic to the schemes described so far is that they form a flat topology (in the sense of non-clustered) with a single TDMA structure to convey node-to-sink traffic following a multi-hop routing scheme. More information about such protocols can be found in [1], [4], [5] and [9].

To avoid the global synchronization requirement imposed by some TDMA techniques, or when multi-hop is not the preferable routing scheme from the point of view of energy consumption, other TDMA-based strategies adopt a cluster organization. Probably the most popular one is Low-Energy Adaptive Clustering Hierarchy (LEACH) [13]. Here, the sensor network is organized into clusters, and intra-cluster communications follow a TDMA scheme rooted at the cluster head, a node within the cluster that centralizes all control operations. Thus, communication is two-hop: from the sensor node to its cluster head, which fuses the data from all nodes within the cluster, and from the cluster head to the sink (LEACH does not specify any particular scheme for this second hop). Since the two distances can be relatively large, especially if the sink is located remotely, the cluster heads are rotated within the network according to random selection. Anyway, LEACH is not well suited for wide area sensor networks because of the average length of the first hop. By considering the residual energy of nodes and their physical distribution throughout the network in the selection of the cluster heads, the Hybrid Energy-Efficient Distributed Clustering (HEED) [14] protocol achieves network lifetimes over 3-4 times larger than LEACH. Finally, an alternative improvement over LEACH is Power Efficient Gathering in Sensor Information Systems (PEGASIS) [15], where each node communicates regularly over a single-hop distance and takes turns transmitting directly to the sink. The whole network forms a chain of communicating nodes, and only one of them becomes the cluster head at a time. The energy consumption is reduced because of the single-hop transmissions and the high degree of data aggregation (each round the data from all nodes are fused into a single packet). Network lifetime achieves a 1-3 improvement over LEACH. 
As stated in the previous section, this paper focuses on TDMAbased proactive sensor networks where multi-hop becomes more energy-efficient than single or two-hop routing schemes. This happens when single-hop distances are relatively large, especially if for such distances the path loss exponent in the radio propagation model becomes larger than 2 (due to multi-path effects). For these flat, non-clustered topologies, a generic TDMA scheme is assumed, with no especial emphasis on the algorithm for slot assignment. Also, the routing scheme is assumed to be static, as it is the case (because of simplicity) of many environmental sensor networks deployed in structured manner. To alleviate the task of most popular nodes, data aggregation is considered and treated in this paper under a very generic framework. To our knowledge, this is the first contribution to a systematic analysis of energy consumption and network lifetime for this kind of scenarios.

\section{ANALYSIS OF LIFETIME}

To proceed with the analysis of energy consumption and lifetime, let us adopt the following definitions, valid for any node $i=1 \ldots N$, where $N$ is the number of nodes in the network:

- $g(i)$ : Number of packets generated by node $i$ per TDMA frame. If node $i$ is a source node, then $g(i) \geq 1$ (but generally $1)$; if it is not a source node, then $g(i)=0$.

- $r(i)$ : Number of packets received by node $i$ during a TDMA frame.

- $t(i)$ : Number of packets transmitted by node $i$ during a TDMA frame. Note that $t(i) \leq r(i)+g(i)$. The specific value depends on the aggregation pattern at node $i$.

- $a(i)$ : Level of aggregation at node $i$. It denotes the number of transmitted packets that node $i$ fuses from others during a TDMA frame. Note that $a(i) \leq t(i)$, because any transmitted packet may result from aggregation or not.

To illustrate these magnitudes, Figure 1 shows an example with a simple network of 14 nodes. The establishment of aggregation patterns on a per-node basis as well as how all this information is used by channel assignment algorithms are out of the scope of this paper.

For the evaluation of the energy wasted in communication, the following radio model can be used [14], where $E_{T}(i), i=1 \ldots N$ denotes the energy consumed by node $i$ when transmitting a packet a distance $d(i)$ and $E_{R}$ is the energy consumed by any node when receiving a packet:

$$
\begin{aligned}
& E_{T}(i)=E_{e} \cdot m+E_{w} \cdot m \cdot d(i)^{f} \\
& E_{R}=E_{e} \cdot m
\end{aligned}
$$

This model becomes appropriate at least for a first step contribution (which focuses on lifetime and not on performance), as it adapts well to different scenarios including not only free space but also multi-path propagation and other impairments. Note from (1a) that the only difference among nodes is due to their communication ranges. The parameters in (1a)-(1b) are defined as follows:

- $E_{e}$ : Energy dissipated by the transceiver circuitry to transmit or receive a single bit.
- $E_{w}$ : Energy radiated to the wireless medium when transmitting a single bit a distance of 1 meter.

- $f$ : Path loss exponent. It depends on the distance and the environment. It usually takes on values between 2 (free space propagation) and 5-6 (multi-path and other effects) [16].

- $m$ : Packet length in bits.

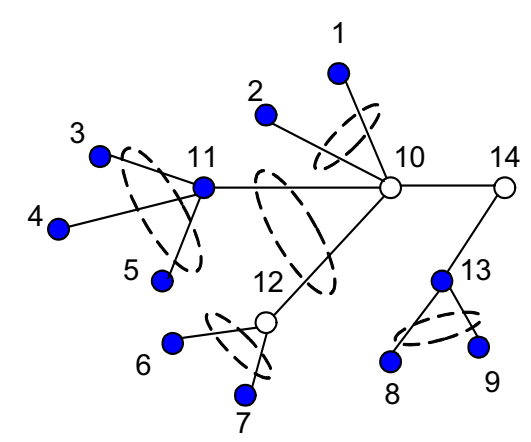

\begin{tabular}{|c|c|c|c|c|}
\hline Node(s) & $g(i)$ & $r(i)$ & $t(i)$ & $a(i)$ \\
\hline \hline $1-9$ & 1 & 0 & 1 & 0 \\
\hline 10 & 0 & 5 & 3 & 2 \\
\hline 11 & 1 & 3 & 2 & 1 \\
\hline 12 & 0 & 2 & 1 & 1 \\
\hline 13 & 1 & 2 & 2 & 1 \\
\hline 14 & 0 & 5 & 5 & 0 \\
\hline
\end{tabular}

Figure 1. Example of hierarchical data aggregation. Only shaded nodes are source nodes. Dashed ellipses indicate data aggregation. In the case of node 10 , it is assumed that data coming from nodes 3-5 are aggregated with data from 6-7, but the packet generated by node 11 is relayed as it is.

As reported in [14] and [16], the distance-dependent component in (1a) depends on the distance itself:

$$
\begin{aligned}
& d \leq d_{0} \Rightarrow E_{w}=E_{f_{s}}, f=2 \\
& d>d_{0} \Rightarrow E_{w}=E_{m p}, f>2
\end{aligned}
$$

Equation (2a) represents free space propagation, which is valid below the reference distance $d_{0}$ (but beyond the far-field distance of the transmitting antenna [16]). Equation (2b) reflects multi-path propagation, which usually takes place at greater distances. $E_{f s}$ and $E_{m p}$ are the particularizations of $E_{w}$ under free space and multi-path conditions respectively.

Finally, let us introduce $E_{\text {startup }}$ as the energy consumed to wakeup a sensor node from sleep state, and $E_{\text {fusion }}$ as the energy consumed to fuse a packet from a set of any number of packets. If $s(i)$ denotes the number of startup operations performed by node $i$ during a TDMA frame (in general, this number depends on the slot assignment scheme, the topology of the network and the location of the node within such topology), the energy consumed by node $i$ per TDMA frame can be expressed as follows:

$$
E(i)=r(i) \cdot E_{R}+t(i) \cdot E_{T}(i)+a(i) \cdot E_{\text {fusion }}+s(i) \cdot E_{\text {startup }}
$$


In general, each node in a proactive sensor network is required to take readings of its local environment at a fixed rate, thus following a periodic sampling pattern. The period is called time interval $T$ and its inverse is the reporting rate $\lambda$. Unless otherwise specified, we can assume that all nodes sample the environment at the same rate (homogeneity), as it is the case of many environmental monitoring applications (anyway, the analysis performed in this paper could be easily extended to the heterogeneous case). The time interval does not necessarily correspond to the duration of a TDMA frame: note that while the time interval is a magnitude specified by the application layer, the duration of TDMA frames is a network parameter that depends on the packet duration, the number of nodes in the network, the algorithm executed for time slot assignment (which, for instance, may or may not perform spatial reusability) and other signaling issues that are related to the MAC layer specification. Thus, expression (3) should be interpreted as the energy consumed by node $i$ during each round of communication (period or time interval), which typically encompasses one or several TDMA frames.

Then, if $B$ denotes the initial energy available at every node in the network (battery), the lifetime $l(i)$ of any node $i$ in number of rounds can be expressed as follows (it is assumed that a node is alive as far as it fulfills the communication requirements of complete rounds):

$l(i)=\left[\frac{B}{E(i)}\right]$

If the packet duration is taken as unit of time (timeslot or simply slot), the normalized lifetime in number of slots obeys the following expression:

$$
l(i)=\left[\frac{B}{E(i)}\right] \cdot \frac{T}{\tau}
$$

We can easily convert magnitudes (4a) and (4b) into real time units by simply multiplying them by the duration of rounds and packets respectively. Note that the application of expressions (4a) or (4b) to the most consuming node in the network yields the network lifetime $L$ (assuming this is defined as the time until first node dies):

$$
L=\left.l(i)\right|_{\min }
$$

Here, $\left.l(i)\right|_{\min }$ would correspond to $\left.E(i)\right|_{\max }$. Another significant metric related with energy consumption and lifetime is the duty cycle. In particular, the duty cycle of node $i$ is given by the quotient between the amount of time this node is active during a communication round and the duration of the round Thus, if $\tau$ denotes the duration of packets and $D C(i)$ the duty cycle of node $i$, this is given by:

$$
D C(i)=\frac{(r(i)+t(i)) \cdot \tau}{T}=\lambda \cdot \tau \cdot(r(i)+t(i))
$$

Specifically, the duty cycle of the node that determines network lifetime will be simply denoted as $D C$. Note that this is a relevant factor, but not necessarily the largest duty cycle in the network: the energy consumption depends not only on the duty cycle, but also on the transmission range - see expressions (1a) and (3).

\section{NUMERICAL EXAMPLE}

To illustrate the previous analysis and to derive the main conclusions of this paper, we can consider the simple large-scale linear network shown in Figure 2, where 100 equally-spaced sensor nodes form a line that covers a distance of $10 \mathrm{~km}$. In order to perform a pessimistic evaluation, we can assume that all nodes are source nodes, that is, they all sense the environment and generate packets, and that data are not aggregated at any point in the network. We can also assume that all nodes generate the same traffic (homogeneous case), quantified as 1 packet every time interval. Then, by numbering nodes successively from left to right (see Figure 2), the following equations hold:

$$
\begin{aligned}
& g(i)=1, \forall i=1 \ldots 100 \\
& r(i)=i-1, \forall i=1 \ldots 100 \\
& t(i)=g(i)+r(i)=i, \forall i=1 \ldots 100 \\
& a(i)=0, \forall i=1 \ldots 100 \\
& d(i)=d \Rightarrow E_{T}(i)=E_{T}, \forall i=1 \ldots 100
\end{aligned}
$$

Under these conditions, the energy consumption model given by (3) takes the following form:

$$
E(i)=(i-1) \cdot E_{R}+i \cdot E_{T}, \forall i=1 \ldots 100
$$

Note that node 100 will determine the lifetime of the network, since this node is responsible for delivering all packets to the base station. Regarding the duty cycle, expression (6) becomes as follows:

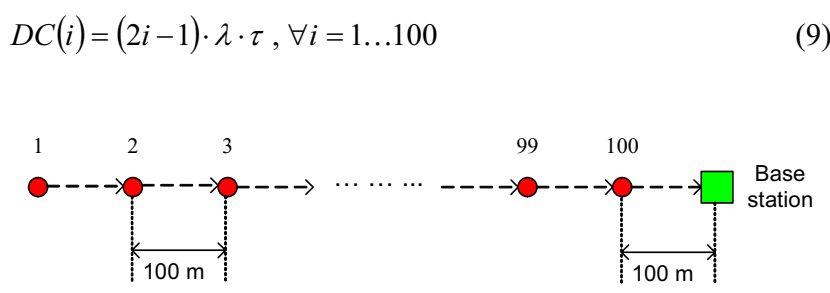

Figure 2. Large-scale linear sensor network.

We can also construct alternative expressions to (8) and (9) by introducing the forwarding factor $\sigma(i), i=1 \ldots N$, as the number of source nodes from which a given node $i$ forwards packets. This variable will be especially useful in the next section. Then, for the linear network of Figure 2, the forwarding factor adopts the following form:

$$
\sigma(i)=i-1, \forall i=1 \ldots 100
$$

Correspondingly, expressions (8) and (9) can be rewritten as follows:

$$
\begin{aligned}
& E(i)=\sigma(i) \cdot\left(E_{R}+E_{T}\right)+E_{T}, \forall i=1 \ldots 100 \\
& D C(i)=(2 \sigma(i)+1) \cdot \lambda \cdot \tau, \forall i=1 \ldots 100
\end{aligned}
$$

From expression (11), we note that the node with largest forwarding factor will determine the lifetime of the network (again node 100).

In order to validate the numerical analysis, simulation results were also obtained. This required the adoption of a particular slot assignment scheme, without detriment of the generality of the 
paper and the results regarding network lifetime. Thus, for simplicity, a DFS scheme without spatial reusability was assumed (see previous section). An observation that deserves attention is that the purpose of simulation here is just to validate the analysis performed in the previous section, hence it becomes rather trivial, because the system under consideration (with the assumptions adopted so far) does not have any random component and it is really deterministic: TDMA frame structure, periodic packet generations and static routing scheme. In fact, results with zero confidence interval were obtained. To achieve them, the radio characteristics shown in Table 2 were considered. Here, the realistic values used in [13] were extended to account for multipath propagation. The 75 meters for the reference distance is common in outdoor environments $[14,16]$. Note also that the startup energy was neglected. Despite this approximation must be verified in every particular scenario, it is quite common at least in first step evaluations, as it does not affect the main results. In addition, a low value of $0.3 \mathrm{~J}$ for the battery was assumed in order to limit the length of the simulation process (throughout this paper different values for the battery will be used for convenience, but this is of secondary importance since network lifetime is quasilinearly proportional to the battery level - see expressions (4a)(4b)).

Table 2. Radio characteristics for the evaluation process.

\begin{tabular}{|l|c|}
\hline$E_{e}$ & $50 \mathrm{~nJ} / \mathrm{bit}$ \\
\hline$E_{f s}$ & $100 \mathrm{pJ} / \mathrm{bit}^{2} \mathrm{~m}^{2}$ \\
\hline$E_{m p}$ & $0.013 \mathrm{pJ} / \mathrm{bit} / \mathrm{m}^{4}$ \\
\hline$f$ (in multi-path) & 4 \\
\hline Reference distance $\left(d_{0}\right)$ & $75 \mathrm{~m}$. \\
\hline$E_{\text {startup }}$ & $\cong 0$ \\
\hline
\end{tabular}

$\square$ Analytical $\square$ Simulation

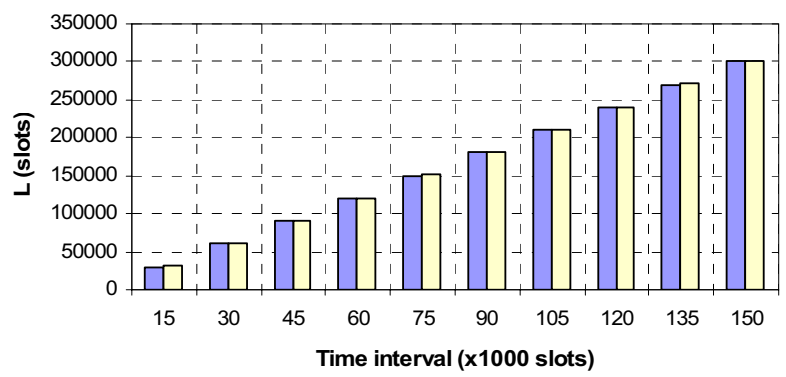

Figure 3. Evolution of network lifetime as the time interval increases $(B=0.3 \mathrm{~J})$.

A first set of results is shown in Figure 3, where the evolution of network lifetime in terms of the time interval is represented. Results from both the analytical and simulation models are provided, showing almost perfect match. the analytical and simulation results obtained for the evolution of network lifetime (the lifetime of node 100) as the time interval increases from 1 to 10 minutes (this is a representative margin for a wide set of environmental monitoring applications). The only difference between analytical and simulation results, hardly appreciable in the figure, is due to the timescale resolution used in the two models: while expression (4b) gives lifetime as the number of timeslots corresponding to an integer number of rounds (formal definition), the energetic analysis in the simulation program is performed on a slot basis for simplicity (slot per slot), so it finally reflects the activity of the most consuming node during a residual number of slots within the last non-complete communication round.

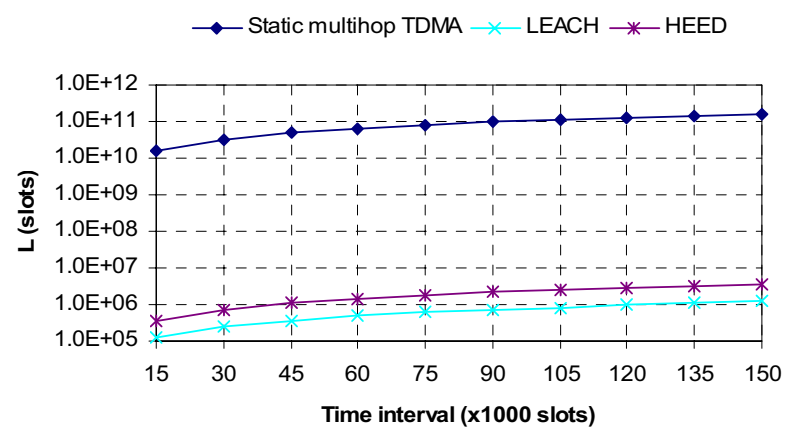

Figure 4. Comparative lifetimes for different proactive protocols and time intervals $(B=150 \mathrm{~kJ})$.

To notice the benefits of a static multi-hop TDMA-based scheme in terms of lifetime, this is compared to other proactive protocols like LEACH or HEED - see Section 2 for details. Then, based also on simulation results (where now systems like LEACH or HEED are no longer deterministic), Figure 4 shows that TDMA largely outperforms the two dynamic protocols for the large-scale environmental network considered here. In fact, in order to obtain significant lifetimes for the dynamic protocols, the battery in this experiment was set to $150 \mathrm{~kJ}$, which is a very large but still realistic value (see [2]). With such a battery, the simulation results shown in Figure 4 were linearly extrapolated from much less computationally-efficient battery values. These results are not surprising, since both LEACH and HEED are more suited to dense randomly-deployed sensor networks, with rather short single-hop distances. Thus, in essence, Figure 4 reflects the appropriateness of multi-hop over single-hop (or two-hop) routing schemes whenever the distance-dependent component in the radio propagation model dominates energy consumption in expression (1a). This happens precisely for large single-hop distances.

The results shown in Figure 4 can be converted into real time units by fixing the duration of packets. A realistic value could be $4 \mathrm{~ms}$., corresponding to packets of $125 \mathrm{~B}$ transmitted at $250 \mathrm{Kbps}$. In this case, a time interval of 15000 slots corresponds to 1 minute. And for a time interval of 6 minutes (90000 slots), the lifetime of a TDMA-based scheme predicted by Figure 4 is approximately 12 years. Then, a realistic value of 1.2 years is finally obtained by switching the energy capacity to a more realistic value of $15 \mathrm{~kJ}$ (instead of $150 \mathrm{~kJ}$ ), as it is the case of Berkeley MICA Motes [2].

Considering from now on the TDMA-based scheme, for $B=15 \mathrm{~kJ}$ and $\tau=4 \mathrm{~ms}$., an interesting result is the evolution of the duty cycle in communication $(D C)$ as a function of the time interval. 
This is shown in Figure 5. Note that the worst value is obviously achieved for the smallest time interval, although it is still quite low (about $1.3 \%$ ). Many other curves can be obtained by varying different configuration parameters and radio characteristics. For instance, another interesting result is the evolution of network lifetime as the number of nodes varies, assuming the linear topology of Figure 2. This is shown in Figure 6, where the time interval has been set to 5 minutes. Figure 7 shows the corresponding evolution of the duty cycle in communication. Note that obviously the performance shown in Figures 6 and 7 degrades as the number of nodes increases, but still satisfactory values are obtained for the worst case (200 nodes), where the lifetime is around 6 months and the duty cycle is less than $0.6 \%$.

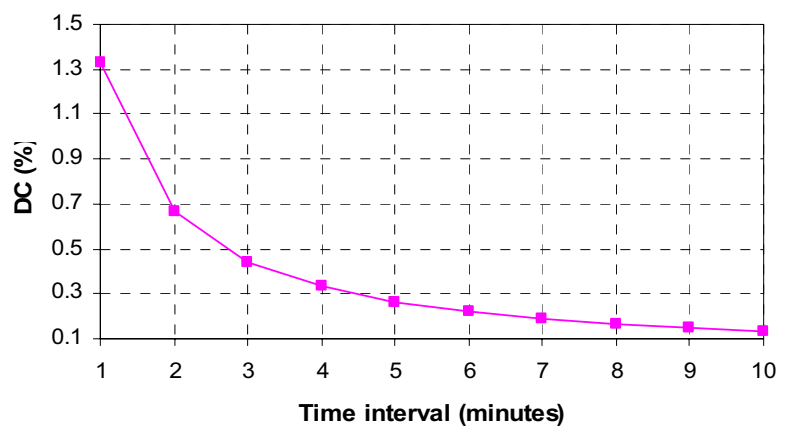

Figure 5. Evolution of the duty cycle in communication as the time interval increases.

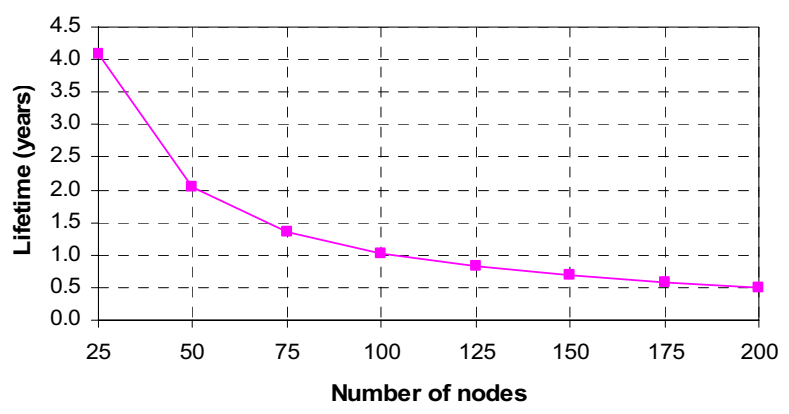

Figure 6. Network lifetime as a function of the number of nodes ( $T=5$ minutes).

\section{INSERTION OF SWITCHING NODES}

In proactive sensor networks, not necessarily all nodes sense the environment and generate packets. For instance, in the BFS/DFSbased scheduling considered in [10], only some nodes are marked as source nodes (typically the outermost ones). This has been taken into account in the general framework presented in Section 3 , through the traffic variable $g(i)$. If it takes on the value 0 for a particular node, this behaves as a pure switching device (what means that its sensing capabilities have been detached or deactivated), and its role in the network is to help source nodes to convey their packets towards the base station. In this sense, in line with the structured deployment assumed in this paper, it is important to locate switching nodes so as to support the most energy-consuming source nodes in the network. These are in general the source nodes with largest transmission ranges, largest forwarding factors or largest reporting rates (in the heterogeneous case).

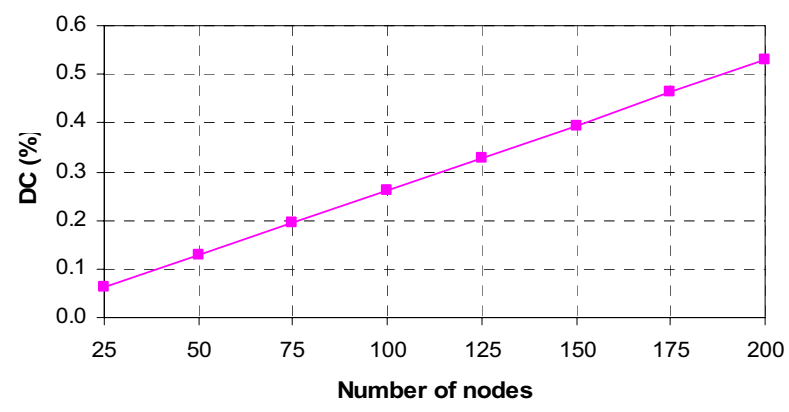

Figure 7. The duty cycle in communication as a function of the number of nodes ( $T=5$ minutes).

In general, planning a network with both source and switching nodes can be decomposed into two stages:

- First, only source nodes are considered, with their locations imposed by the user/application level. Then, the resulting topology and the routing algorithm determine in turn the routing table and hence the forwarding factor for each node.

- Next, based on the transmission range and forwarding factor of each source node, the locations of switching nodes can be determined in order to satisfy a certain requirement on network lifetime. The products offered by some manufacturers allow for an easy and cost-effective implementation of such strategy, since these products follow a modularized architecture where the sensing module can be detached from the mote to convert the node into a pure switching device.

The whole process can be implemented in a semi-automated way by forcing source nodes to determine their routes and forwarding factors (by sending probe packets), and to deliver this global information to the base station, where a switching node deployment is obtained. This is then performed manually. In a more advanced version, the process could be completely automated by using mobile nodes (robots with application and/or network layer functionalities) [1, 4]. Whatever alternative is adopted, the two stages follow essentially the process described in [4] for structured deployment, where some nodes are inserted just to achieve full connectivity.

A motivating example for switching node insertion is again a linear network, like the one depicted in Figure 2, but with only 5 nodes covering a distance of half kilometer (the issue of scalability will be treated in the next section). For simplicity, let us measure lifetime in number of rounds and set the battery again to 0.3 Joules. The rest of data are taken from Table 2. An interesting analysis is the evolution of node lifetime in terms of the position of the node. As it is shown in Figure 8, the resulting curve reveals huge differences: when node 5 becomes exhausted, node 1 could survive for about 5 times more!

Then, a way of increasing the lifetime of the network and achieving a more fair distribution of energy consumption is by 
inserting pure switching nodes in the links between source (regular) nodes. A systematized procedure is adopted here, where switching nodes are uniformly spaced between regular nodes. Figure 9 illustrates this idea for a general setup consisting of two adjacent regular nodes with arbitrary forwarding factors. Note that by inserting $n(i)$ switching nodes, the upstream regular node (node $i$ ) needs only to transmit a distance $n(i)+1$ times smaller, and consequently the energy it consumes to transmit a packet becomes $(n(i)+1)^{f}$ times smaller - see expression (1a). Such a distance reduction becomes especially significant when it is accompanied by a reduction of the path loss exponent - see expressions (2a)-(2b).

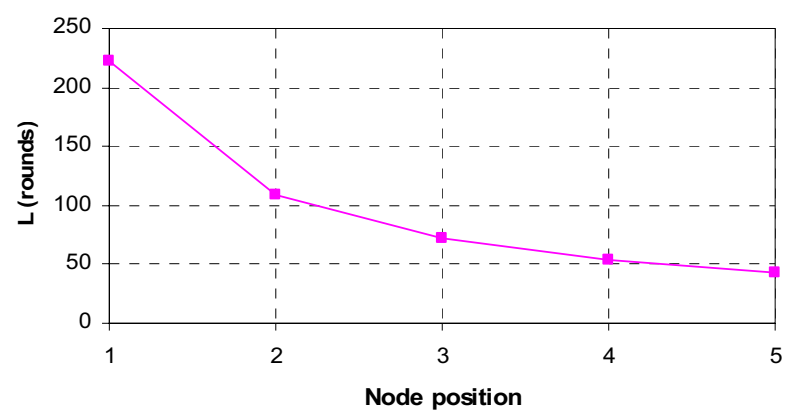

Figure 8. The lifetime of nodes in terms of their position $(B=$ 0.3 J).

In Figure 9, it has been assumed that the inserted nodes are exclusively dedicated to link $(i, j)$ (pessimistic criterion). Thus, it is enough to index them through the upstream node index $i$ only. Several additional observations deserve attention:

- First, note also that the presence of switching nodes does not alter the forwarding factor of the downstream node $j$, obviously because switching nodes do not generate packets.

- All switching nodes for a given upstream regular node $i$ have the same forwarding factor.

- Given two adjacent regular nodes $i$ and $j$ with respective forwarding factors $\sigma(i)$ and $\sigma(j)$, we can establish the following relationships, where $\sigma(i, k), i=1 \ldots N, k=1 \ldots n(i)$ is the forwarding factor of the switching node located at position $k$ in the link $(i, j)$ :

$$
\sigma(i, k)=\sigma(i)+1 \leq \sigma(j), \forall i, j=1 \ldots N, k=1 \ldots n(i)
$$

Figure 9 represents any pair of adjacent regular nodes in a network of arbitrary topology. However, the set constituted by the upstream node $i$ and all subsequent inserted nodes can also be viewed as a linear sub-network like the one depicted in Figure 2, with the downstream node $j$ behaving as a virtual base station. Then, assuming that the energy model given by equation (11) holds, we can extend it to any inserted node in Figure 9 as follows:

$$
E(i, k)=\sigma(i, k) \cdot\left(E_{R}+\cdot E_{T}\right), \forall i=1 \ldots N, \forall k=1 \ldots n(i)
$$

Here, $E(i, k)$ denotes the energy consumed by any node inserted in the link from node $i$ during a communication round. Then, by combining expressions (13) and (14) we obtain the following result:

$$
E(i, k)=E(i)+E_{R}, \forall i=1 \ldots N, \forall k=1 \ldots n(i)
$$

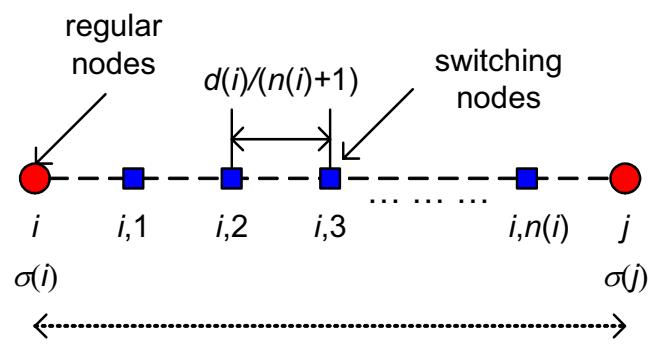

$d(i)$

Figure 9. Insertion of switching nodes in the link between two regular adjacent nodes.

This means that the lifetime of inserted nodes is always slightly smaller than the lifetime of the corresponding upstream node. We cannot say anything with regards to the lifetime of the downstream node, because this depends on the transmission range of such node, which is not specified in the insertion model represented in Figure 9.

To illustrate the process of node insertion, let us continue with the 5 -node linear network and consider the objective of enhancing its lifetime by a factor 5 (again for the data listed in Table 2). This means that the lifetime of all nodes in the network (including the inserted nodes) must be of at least $5 \cdot 43=215$ rounds, where 43 is the current network lifetime (given by node 5 - see Figure 8 ). By inspecting the data supporting Figure 8, we can observe that node 1 already satisfies this requirement, so it is not necessary to insert switching nodes in its link to node 2. Contrarily, nodes 2-5 require some enhancement. Then, proceeding from node 2 to node 5 and by inserting the minimum number of switching nodes necessary to satisfy the 215-round lifetime requirement, we obtain a new lifetime curve shown in Figure 10. In particular, one node has been inserted in every outgoing link from node 2 to node 4 , and two nodes have been inserted between node 5 and the base station (see Figure 11). Figure 10 also includes the original lifetime curve as well as the curve corresponding to inserted nodes (indexed by the positions of the associated regular nodes). Note that certainly we have duplicated the number of nodes in the network, which might not look efficient, but network lifetime has increased by a factor 5 ! We can also see the effectiveness of such enhancement from the point of view of the energy capacity: by doubling the number of nodes, which means doubling the total energy available, we have increased lifetime by a factor 5; however, if we had simply doubled the energy capacity (battery) of nodes, lifetime would have only doubled too! In general, the reason is that the component of radiated power in the radio propagation model - expression (1a), yields to significant reductions when the distance between two adjacent nodes is also reduced. This is in turn due to the effects of the path loss exponent, which typically varies between 2 and 6 [16]. For instance, when the distance between adjacent nodes is divided by 2 , the radiated power is reduced a factor that varies between 4 (free space conditions) and 32 (strong multi-path effects). 
Obviously, this reduction is not linearly reflected in the node lifetime because of the distance-independent component of the energy model, which attenuates all variations. Nevertheless, as the previous example shows, a network lifetime 5 times larger is achieved.

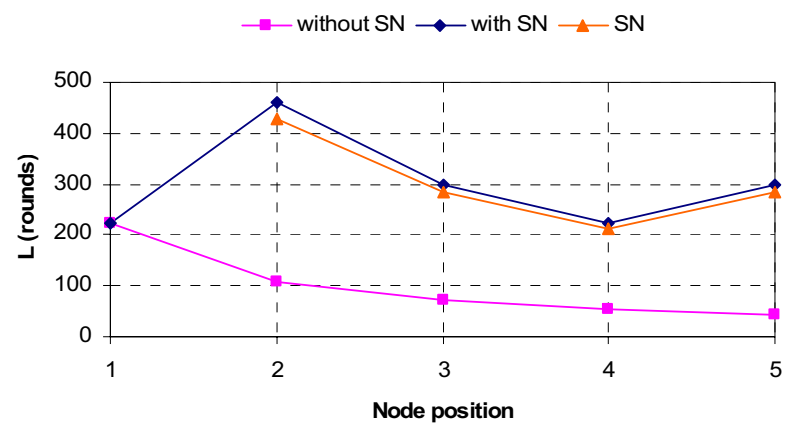

Figure 10. Lifetime curves with and without switching nodes for the 5-node network (SN stands for switching node). Lifetime is improved by a factor $5(B=0.3 \mathrm{~J})$.

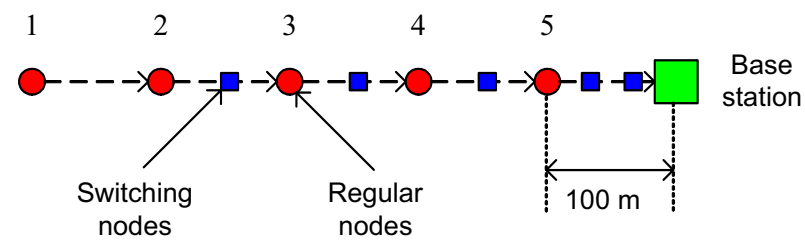

Figure 11. Switching nodes inserted in the links between regular nodes to enhance the lifetime of the 5-node network by a factor $5(B=0.3 \mathrm{~J})$.

To quantify the process of switching node insertion, let us define $n$ as the number of switching nodes that must be inserted in the link from an upstream node with generic forwarding factor $\sigma$ and initial transmission range $d$, in order to guarantee a network lifetime $L$ in number of rounds. By combining expressions (1a), (1b), (4) and (11), we can derive the following expression for $n$, which is independent of the topology of the network:

$n=n(\sigma, d, L)=\left\lceil\left(\frac{L \cdot(\sigma+1) \cdot m \cdot E_{w} \cdot d^{f}}{B-L \cdot(2 \sigma+1) \cdot m \cdot E_{e}}\right)^{1 / f}-1\right\rceil$

In the evaluation of this formula, it must be taken into account that actually $E_{w}=E_{w}(d, n)$ and $f=f(d, n)$ because of (2a) and (2b).

By applying equation (16) to the 5-node linear sensor network (with the data listed in Table 2), we can obtain the evolution of the total amount of nodes that must be inserted so as to guarantee a given lifetime amplification. This result is represented in Figure 12 , where the number of nodes to be inserted is expressed as a percentage relative to the number of regular nodes (5).

Note that the configuration shown in Figure 11 corresponds to the amplification factor 5 in Figure 12. Note also that with a $200 \%$ increase in the number of nodes (thus from 5 to 15) the lifetime becomes 9 times larger. In general, the curve represented in Figure 12 exhibits an excellent balance between lifetime increase and percentage of node insertion, where the selection of the final configuration would possibly depend on other factors. For instance, the transmission range of any device should stay within the Fraunhofer region [16] in order to avoid near-field effects. Another factor is obviously the specific cost of switching nodes.

In general, the behavior shown in Figure 12 depends on the number of nodes, the original transmission range and the specific values of the components of the radio propagation model, specially the relationship between the distance-dependent and the distance-independent terms. Anyway, the profile shown in Figure 12 can be considered as representative of TDMA-based networks with structured deployment and small number of nodes. Next, we discuss scalability issues.

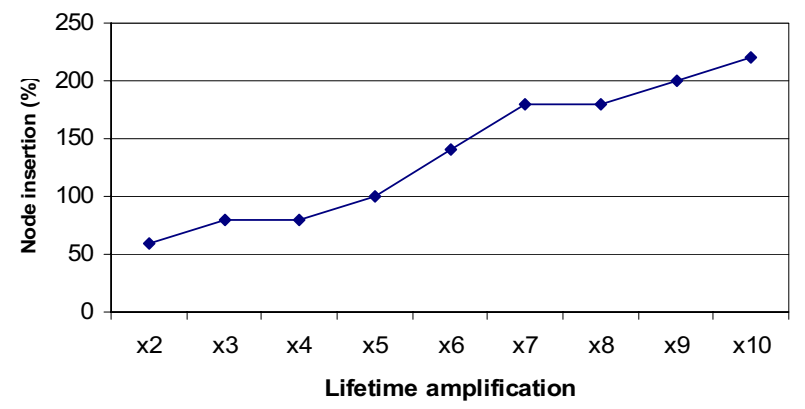

Figure 12. Number of inserted nodes in percentage relative to the number of regular nodes (5) as a function of the lifetime amplification factor (where the lifetime without node insertion is 43 rounds and $B=0.3 \mathrm{~J}$ ).

\section{SCALABILITY ISSUES}

The purpose of this section is to investigate the scalability of the switching node insertion mechanism described so far. More specifically, the focus is on scalability regarding geographical coverage and scalability regarding number of nodes.

\subsection{Scalability in Geographical Coverage}

To analyze the scalability in geographical coverage, let us first consider Figure 13 where the lifetime of nodes has been represented in terms of their position for different values of the number of switching nodes inserted per link. Again the linear network constituted by 5 nodes with the data listed in Table 2 has been considered. The battery is $0.3 \mathrm{~J}$. Note in the figure that each curve corresponds to the same number of switching nodes inserted per link, although for a given lifetime requirement only the nodes with largest forwarding factor would really need some enhancement (see Figure 11). In other words, the curves in Figure 13 represent upper bounds on the intensity of the node insertion process, and thus in a real scenario a combination of several curves rather than a single one would actually be configured. For some of these configurations, the resulting network lifetime might not correspond to the node with largest forwarding factor, but still this node would provide a reliable estimate of lifetime. Thus, aside from implementation and configuration issues, we can take 
the node with largest forwarding factor as fully representative to provide that estimate. Accordingly, a convincing proof of scalability in coverage is given in Figure 14, where the lifetime of a node with forwarding factor 4 (the node with largest forwarding factor in the 5-node linear network) is represented in terms of the number of switching nodes inserted, for different values of the initial transmission range (the transmission range without node insertion). For this magnitude, a margin between 50 and 300 meters has been considered, which is fully representative of sensor networks with structured deployment.

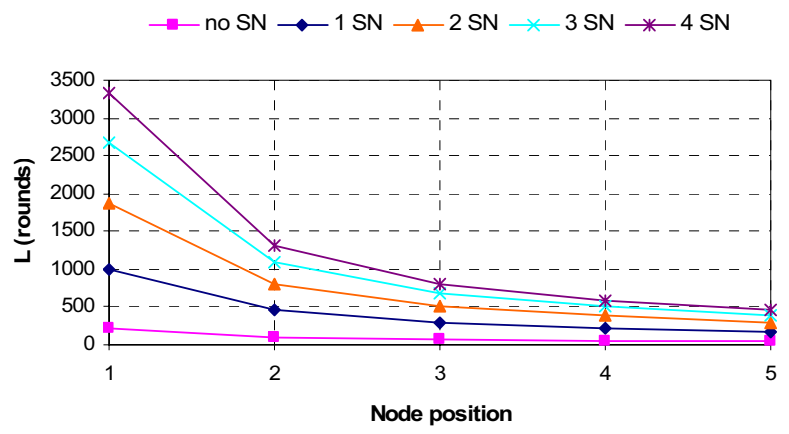

Figure 13. The lifetime of nodes as a function of their position for different values of the number of switching nodes inserted per link $(B=0.3 \mathrm{~J})$.

A detailed analysis of Figure 14 reveals that the lifetime increase is larger for larger initial transmission ranges and from smaller amounts of switching nodes inserted. This is mathematically explained by equation (1a). Nevertheless, there is a wide set of points in Figure 14 for which node insertion becomes cost effective. On the other hand, the amount of points with significant lifetime enhancement decreases as the value of the forwarding factor increases. This is treated next in more detail.

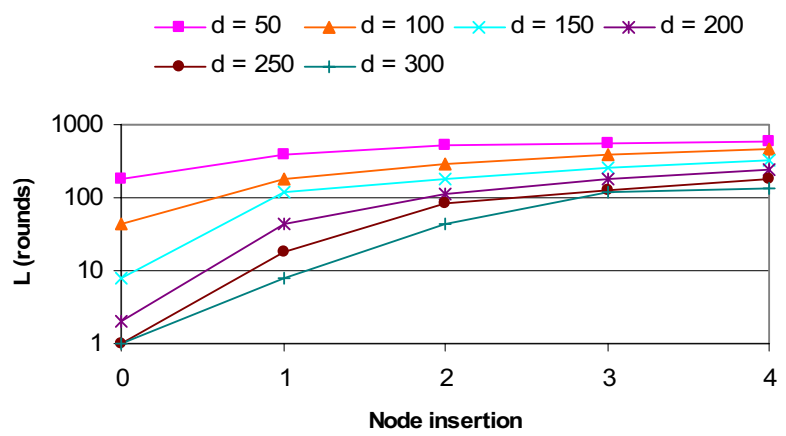

Figure 14. Node lifetime as a function of the number of switching nodes inserted for different values of the initial transmission range (in meters). A node with forwarding factor 4 has been considered.

\subsection{Scalability in Number of Nodes}

To analyze the scalability in number of nodes, we can fix the initial transmission range (for instance 200 meters) and vary the forwarding factor of the node directly connected to the base station, which is a direct consequence of modifying the number of nodes (again in our small-scale linear network). Figure 15 shows the results. Two observations deserve attention:

- For a given forwarding factor, as the number of inserted nodes increases, the lifetime improvement decreases in relative terms.

- For a given number of switching nodes inserted, the lifetime improvement becomes more significant for smaller forwarding factors.

In general, this means that the scalability in size is small. Thus, the case of networks with arbitrarily high number of nodes is treated next.

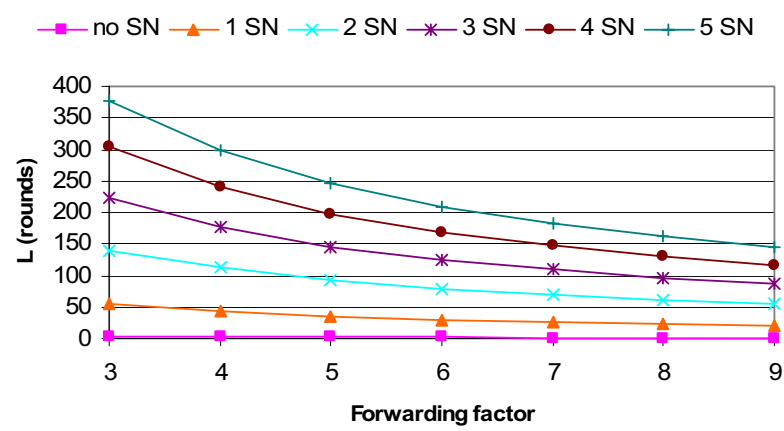

Figure 15. Evolution of node lifetime as the forwarding factor increases for different levels of node insertion.

\subsection{What Happens with Larger Number of Nodes?}

In general, a sensor network may contain hundreds or even thousands of nodes forming arbitrary topologies, thus making impractical the introduction of switching nodes as a unique mechanism for lifetime enhancement. However, if such a mechanism is combined with other techniques like data aggregation $[4,5,9,17]$, very cost effective solutions can be achieved. In general, the idea consists of applying some aggregation algorithm to the whole network in order to limit the forwarding factor of nodes to a certain maximum value, and then inserting switching nodes especially in the links from the regular nodes with largest transmission ranges and/or largest forwarding factors. Just to illustrate such a basic idea, let us consider the sensor network shown in Figure 16, which consists of 20 nodes forming a routing tree rooted at the base station. In this figure, it has been assumed that all nodes are source nodes ( $g(i)=1, \forall i=1 \ldots 20)$ and no aggregation is initially performed $(a(i)=0, \forall i=1 \ldots 20)$. Note that the forwarding factor increases as the position of the node gets closer to the base station. In this scenario, the insertion of switching nodes as a unique mechanism for enhancing lifetime would not be practical because of the large forwarding factor corresponding to some nodes. However, let us now assume that some data aggregation is performed, which 
basically consists of fusing the packets coming from upstream nodes that are very close to each other. The result is shown in Figure 17. Now the insertion of switching nodes in the links from the regular nodes with largest transmission ranges and/or forwarding factors becomes more practical, as the new scenario is closer to the parameter configuration considered in the previous section.

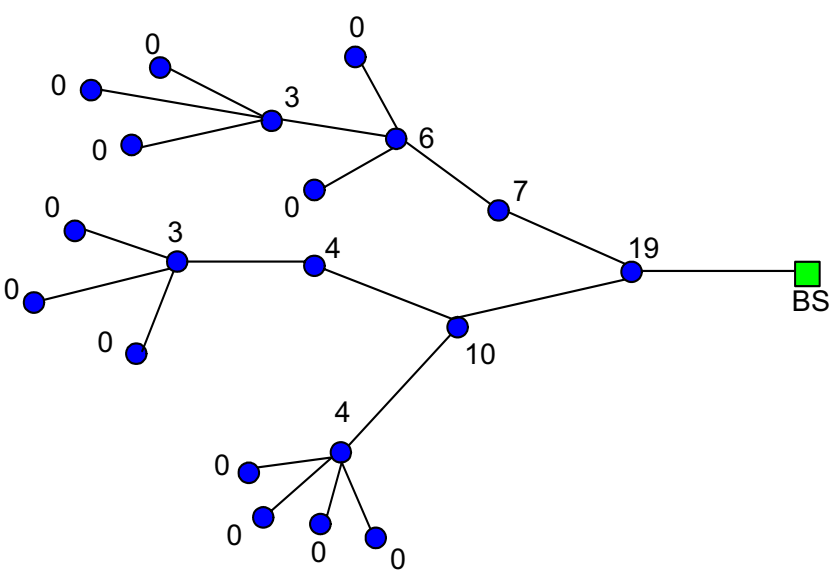

Figure 16. A sensor network with tree topology where all nodes generate packets and no data aggregation is performed.

When analyzing the lifetime of the new configuration, it must be taken into account that a node performing data aggregation still receives all packets from its upstream nodes, and that the energy savings is mainly achieved by the fact of having reduced the number of packets to be transmitted. From a more mathematical point of view, we can recall expression (3), where the component due to the startup operations can again be neglected:

$E(i) \cong r(i) \cdot E_{R}+t(i) \cdot E_{T}(i)+a(i) \cdot E_{\text {fusion }}$

By making some manipulations, this expression can be rewritten in the following way:

$$
E(i) \cong(t(i)-1) \cdot\left[\frac{r(i)}{t(i)-1} \cdot E_{R}+\frac{a(i)}{t(i)-1} \cdot E_{\text {fusion }}+E_{T}(i)\right]+E_{T}(i)
$$

Expression (18) can be viewed as a special case of (11) by identifying the forwarding factor with $t(i)-1$, which corresponds to the way this parameter has been interpreted in Figure 17. On the other hand, the complete identification of expressions (11) and (18) requires the definition of the equivalent receiving energy $E_{R}^{e q u i}$ as follows:

$E_{R}^{\text {equi }}=\frac{r(i)}{t(i)-1} \cdot E_{R}+\frac{a(i)}{t(i)-1} \cdot E_{\text {fusion }}$

Also note that $E_{R}^{e q u i}>E_{R}$, but data aggregation is still recommendable because the reduction of the energy cost of transmitting is usually much larger than the increase represented by equation (19). As stated before, once data aggregation is performed, the mechanism of node insertion can be applied. This strategy can be extended to sensor networks with larger amounts of nodes and arbitrary topologies, which obviously will require more intensive data aggregations. In addition, this could be performed in the completely automated version of the process. However, the detailed treatment of these aspects has been left for further research, as this paper represents a first step contribution.

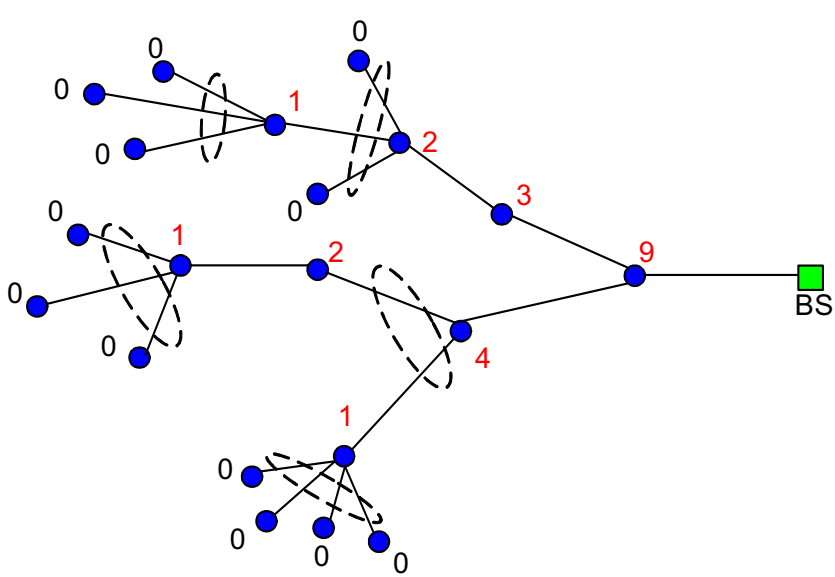

Figure 17. Use of data aggregation to reduce the forwarding factor of nodes (the new values are shown in red). Once performed, node insertion becomes more practical.

\section{CONCLUSIONS}

In this paper, a systematic approach to the problem of lifetime planning for TDMA-based proactive sensor networks with structured deployment has been introduced. This is the case of many environmental networks, where some variable is regularly monitored for scientific study or for taking appropriate control actions (preventive mechanism). For simplicity, it is common that the data generated by these networks follow predetermined paths, giving rise to static routing schemes. In addition, these schemes are typically multi-hop, since single-hop distances in structured deployments are not necessarily small. Thus, in order to reduce the severe effects of the path loss exponent in the radio propagation model, a technique consisting of inserting pure switching nodes has been proposed. The major benefits of this technique take place for small size networks with large single-hop distances. For larger networks, the technique must be combined with other lifetime enhancing mechanisms, like data aggregation. However, the detailed analysis of these combined strategies has been left for further research, as this paper represents a first step contribution.

\section{ACKNOWLEDGMENTS}

This work is supported in part by the MCyT (Spanish Ministry of Science and Technology) under contract TIC2006-02265.

\section{REFERENCES}

[1] Cordeiro, C. de M. and Agrawal, D. P. Ad Hoc and Sensor Networks. Theory and Applications. World Scientific Publishing, 2006.

[2] Zhao, F. and Guibas, L. Wireless Sensor Networks. An information processing approach. Elsevier, 2004. 
[3] Akyildiz, I. F., Su, W., Sankarasubramaniam, Y and Cayirci, E. Wireless sensor networks: a survey. Computer Networks, 38, 4 (2002).

[4] Krishnamachari, B. Networking Wireless Sensors. Cambridge University Press, 2005.

[5] Stojmenovic, I. Handbook of Sensor Networks. Algorithms and Architectures. Wiley, 2005.

[6] FAO (2006). Global Forest Resources Assessment 2005 Report on fires in South American Region. Fire Management Working Paper 5. www.fao.org/forestry/site/fire-alerts/en

[7] Ramaswami, R. and Parhi, K. K. Distributed scheduling of broadcasts in a radio network. In Proceedings of IEEE INFOCOM (April 1989).

[8] Sohrabi, K., Gao, J, Ailawadhi, V. and Pottie, G. J. Protocols for self-organization of a wireless sensor network. IEEE Personal Communications, 7, 5 (Oct. 2000).

[9] Karl, H. and Willig, A. Protocols and Architectures for Wireless Sensor Networks. Wiley, 2005.

[10] Arisha, K. A., Youssef, M. A. and Younis, M. F. Energyaware TDMA based MAC for sensor networks. In Proceedings of IEEE Workshop on Integrated Management of Power Aware communications, Computing and Networking (IMPACCT) (May 2002).
[11] Conner, W. S., Chhabra, J., Yarvis, M. and Krishnamurthy, L. Experimental evaluation of synchronization and topology control for in-building sensor network applications. In Proceedings of ACM WSNA (Sept. 2003).

[12] Rajendran, V., Obraczka, K. and Garcia-Luna-Aceves, J. J. Energy-efficient, collision-free medium access control for wireless sensor networks. In Proceedings of ACM Sensys (Nov. 2003).

[13] Heinzelman, W. B., Chandrakasan, A. P. and Balakrishnan, $\mathrm{H}$. An application-specific protocol architecture for wireless microsensor networks. IEEE Transactions on Wireless Communications, 1, 4 (Oct. 2002).

[14] Younis, O. and Fahmy, S. Distributed clustering in ad-hoc sensor networks: a hybrid, energy-efficient approach. In Proceedings of IEEE Infocom (2004)

[15] Lindsey, S. and Sivalingam, K. M. Data gathering algorithms in sensor networks using energy metrics. IEEE Transactions on Parallel and Distributed Systems, 13, 9 (2002).

[16] Rappaport, T. S. Wireless Communications. Principles and Practice. Prentice-Hall, 2002 (Second Edition)

[17] Raghavendra, C. S., Sivalingam, K. M. and Znati, T. Wireless Sensor Networks. Springer, 2004. 